\title{
Measurement of cortical porosity of the proximal femur improves identification of women with nonvertebral fragility fractures
}

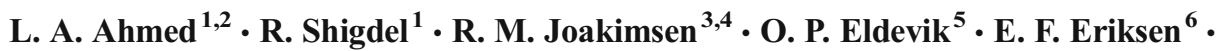 \\ A. Ghasem-Zadeh ${ }^{7} \cdot$ Y. Bala ${ }^{7} \cdot$ R. Zebaze ${ }^{7} \cdot$ E. Seeman ${ }^{7} \cdot$ Å. Bjørnerem ${ }^{1}$
}

Received: 12 November 2014 / Accepted: 23 March 2015 / Published online: 16 April 2015

(C) The Author(s) 2015. This article is published with open access at Springerlink.com

\begin{abstract}
Summary We tested whether cortical porosity of the proximal femur measured using StrAx1.0 software provides additional information to areal bone mineral density (aBMD) or Fracture Risk Assessment Tool (FRAX) in differentiating women with and without fracture. Porosity was associated with fracture independent of aBMD and FRAX and identified additional women with fractures than by osteoporosis or FRAX thresholds.

Introduction Neither aBMD nor the FRAX captures cortical porosity, a major determinant of bone strength. We therefore tested whether combining porosity with aBMD or FRAX improves identification of women with fractures.

Methods We quantified femoral neck (FN) aBMD using dualenergy X-ray absorptiometry, FRAX score, and femoral subtrochanteric cortical porosity using StrAx1.0 software in
\end{abstract}

Å. Bjørnerem

ashild.bjornerem@uit.no

1 Department of Health and Care Sciences, UiT-The Arctic University of Norway, Tromsø, Norway

2 Institute of Public Health, College of Medicine and Health Sciences, United Arab Emirates University, Al Ain, United Arab Emirates

3 Department of Clinical Medicine, UiT-The Arctic University of Norway, Tromsø, Norway

4 Department of Internal Medicine, University Hospital of North Norway, Troms $\varnothing$, Norway

5 Department of Radiology, University Hospital of North Norway, Tromsø, Norway

6 Department of Clinical Endocrinology, Oslo University Hospital, Oslo, Norway

7 Endocrine Centre, Austin Health, University of Melbourne, Melbourne, Australia
211 postmenopausal women aged 54-94 years with nonvertebral fractures and 232 controls in Tromsø, Norway. Odds ratios (ORs) were calculated using logistic regression analysis.

Results Women with fractures had lower FN aBMD, higher FRAX score, and higher cortical porosity than controls (all $p<0.001)$. Each standard deviation higher porosity was associated with fracture independent of FN aBMD (OR 1.39; $95 \%$ confidence interval 1.11-1.74) and FRAX score (OR 1.58; $1.27-1.97)$ in all women combined. Porosity was also associated with fracture independent of FRAX score in subgroups with normal FN aBMD (OR 1.88; 1.21-2.94), osteopenia (OR $1.40 ; 1.06-1.85)$, but not significantly in those with osteoporosis (OR 1.48; 0.68-3.23). Of the 211 fracture cases, only 18 women $(9 \%)$ were identified using FN aBMD T-score $<-2.5$, 45 women $(21 \%)$ using FRAX threshold $>20 \%$, whereas porosity $>80$ th percentile identified 61 women $(29 \%)$. Porosity identified $26 \%$ additional women with fractures than identified by the osteoporosis threshold and $21 \%$ additional women with fractures than by this FRAX threshold.

Conclusions Cortical porosity is a risk factor for fracture independent of aBMD and FRAX and improves identification of women with fracture.

Keywords Bone mineral density $\cdot$ Cortical porosity $\cdot$ FRAX · Nonvertebral fractures

\section{Introduction}

Bone fragility is a public health problem due to the accompanying increased morbidity, mortality, and financial costs resulting from fractures, in particular, nonvertebral and hip fractures $[1,2]$. To reduce the burden of disease cost- 
effectively, methods are needed to identify persons at risk for fracture in need of treatment and persons at low risk to avoid unnecessary treatment. The most common approach used to assess fracture risk is measurement of femoral neck (FN) areal bone mineral density (aBMD) using dual-energy X-ray absorptiometry (DXA) [3]. Although a lower aBMD is associated with higher fracture rates, most fractures occur in women without osteoporosis as defined by the diagnostic threshold of an FN aBMD T-score of 2.5 or more standard deviations (SD) below the young normal mean $[4,5]$.

To address this lack of sensitivity, the World Health Organization (WHO) developed the Fracture Risk Assessment Tool (FRAX) which calculates the 10-year probability of a major osteoporotic fracture based on a combination of clinical risk factors and FN aBMD. This tool has improved the identification of persons at risk of fracture in some, but not all, cohorts [6-8]. Both aBMD and FRAX identify persons at risk, but they do not take into account the cortical porosity, which is an important determinant of bone strength $[9,10]$.

Although both cortical and trabecular bone contribute to bone strength, $80 \%$ of the skeleton is cortical, $70 \%$ of all appendicular bone loss is cortical, and $80 \%$ of fractures in women over 65 years of age are nonvertebral [11, 12]. Cortical bone loss is the result of unbalanced and accelerated intracortical remodeling initiated at points upon the intracortical surface formed by the many Haversian canals traversing it. As more bone is resorbed than replaced at each remodeling site, focal enlargement of the canals occurs, adjacent canals coalesce forming giant pores when viewed in cross section [13-15]. Just as the increase in porosity increases bone fragility, several studies now demonstrate that treatment reduces porosity $[16,17]$. Women with hip fracture have thinner and more porous cortices than controls, and they have giant pores [13-15]. Thus, as cortical bone is an important determinant of bone strength [9], its deterioration should be quantified in assessing fracture risk.

Studies using high-resolution peripheral quantitative computed tomography (HR-pQCT) images of the distal radius and tibia and digital X-ray radiogrammetry of metacarpal bone have demonstrated associations between cortical porosity and fracture [18-20]. As HR-pQCT is not widely available, we examined whether cortical porosity quantified in vivo using standard CT technology, which is available in most institutions, can be used to acquire images of the cortex and its porosity. We tested the following hypotheses: (i) measurement of cortical porosity at the proximal femur, a common site of the most serious fragility fracture, is associated with nonvertebral fracture, (ii) the association between porosity and fracture is independent of aBMD and FRAX, (iii) combining measurements of porosity with aBMD or FRAX improves identification of women with fractures than any one risk assessment method alone.

\section{Materials and methods}

\section{Study design and participants}

These hypotheses were tested in the Tromsø Study, a singlecenter population-based health study in Norway, which conducted six surveys in 1974, 1979-1980, 1986-1987, 1994 1995, 2001-2002, and 2007-2008, respectively [21]. During the Tromsø 4 survey in 1994-1995, all 37,558 eligible inhabitants in Tromsø over 24 years were invited and 27,158 subjects $(72 \%)$ participated. All their nonvertebral fractures were registered from the x-ray archives of the University Hospital of North Norway, Tromsø between Tromsø 4 (1994-1995) and 1 January 2010 [22, 23].

In a nested case-control design, we identified 1250 women participating in Tromsø 4, who suffered a fracture at the hip, wrist, or proximal humerus after the age of 50 years, during the 15-year registry of fractures (1994-1995 to 2010). We invited all 760 who still were alive and living in Tromsø. We recruited 264 fracture cases after excluding premenopausal women, women with bisphosphonate exposure for osteoporosis, pathological fractures or women with hip prostheses, or metal screws in the hip region, because metal on one side can make noise in the CT images of both sides, and therefore many women with hip fractures could not be included. Agematched fracture-free women were randomly selected among the Tromsø 4 participants, 1186 were invited, and after applying the same exclusion criteria, 260 controls were recruited. Of these 524 participants, we excluded 15 currently receiving hormone replacement therapy and 66 with movement artifacts during CT scanning. This left 443 women in the final analyses: 232 controls and 211 fracture cases (4 hip, 181 wrist, and 26 proximal humeral). The median time since their index fracture was 6.6 years. All measurements were performed from November 2011 through January 2013. All participants gave written informed consent. The study was approved by the Regional Committee of Research Ethics and the Norwegian Data Inspectorate and was conducted in accordance with the World Medical Association Declaration of Helsinki.

\section{Procedures}

A self-administered questionnaire included information concerning all fractures after the age of 50 years, diseases, use of medication, and lifestyle. Height and weight were measured in light clothing without shoes. Total hip and FN aBMD were measured at the nondominant side using DXA (GE Lunar Prodigy, Lunar Corporation, Madison, WI, USA), and at the opposite side in those with hip fracture at the nondominant side, and the coefficients of variation (CV) were 1.2 and $1.7 \%$, respectively [24]. The women were categorized into those with normal FN aBMD, osteopenia, and osteoporosis using the WHO classification, 
and the T-score was based on the National Health and Nutrition Examination Survey (NHANES III) reference [24].

The 10-year probability of a major osteoporotic fracture was calculated using the country-specific WHO FRAX algorithm for Norwegian women, including age, height, weight, parental hip fracture, rheumatoid arthritis, smoking, alcohol intake, glucocorticoid therapy, and FN aBMD [6-8]. The nonvertebral fractures used as inclusion criteria of this study were not included as "previous" fracture in the calculation of the FRAX score, as the aim was to assess 10 -year probability of fracture before the event, not the probability of fracture after this event. We did include other fractures that occurred prior to the index fracture in the FRAX score, and these fractures were identified from information in questionnaires at the Tromsø 4 and the current study. FRAX is designed to be used with and without FN aBMD.

CT scans (Siemens Somatom Sensation 16, Erlangen, Germany) of the nondominant hip were performed at the Department of Radiology, University Hospital of North Norway. The CT machine had an in-plane resolution of $0.74 \mathrm{~mm}$, the slice thickness was $0.6 \mathrm{~mm}$, the hip was scanned from just above the femoral head to $2 \mathrm{~cm}$ below the lesser trochanter (Fig. 1), and the exposure dose of radiation was $\sim 1.5 \mathrm{mSv}$. Images were analyzed in Melbourne, Australia, using StrAx1.0 software [25]. As cortices are thin at the proximal femur (femoral head, neck, and trochanter), analyses were confined to a region of interest (ROI) where the cortices are thicker. This 3.7-mm subtrochanteric region starts at tip of the lesser trochanter.

The subtrochanteric region within the ROI in low resolution CT images was segmented into the compact-appearing cortex, transitional zones, and trabecular compartment using StrAx1.0, a non-thresholding method which automatically selects attenuation profile curves as reported using HR-pQCT images [25]. Porosity within each cortical compartment was also quantified automatically throughout the ROI, and similarly in CT images as reported in HR-pQCT images [25]. Local bone edges were identified as the beginning and the end of the rising and falling S-shaped portions of the profile curve enabling the delineation of the compartments [25]. Compartments were segmented by analyzing $\sim 3600$ consecutive overlapping profiles around the perimeter of each crosssectional slice. The density profile curve produced had two plateaus: one corresponding to the compact-appearing cortex and one corresponding to the trabecular compartment. Between these plateaus was a descending S-shaped curve or transition between the two plateaus. This was the transitional zone. The density profile curve was expressing the mineralized bone area as the percentage of total area within each column.

Porosity presented in this study is the average void volume fraction summed using all voxels within the total cortex (compact-appearing cortex, outer and inner transitional zones
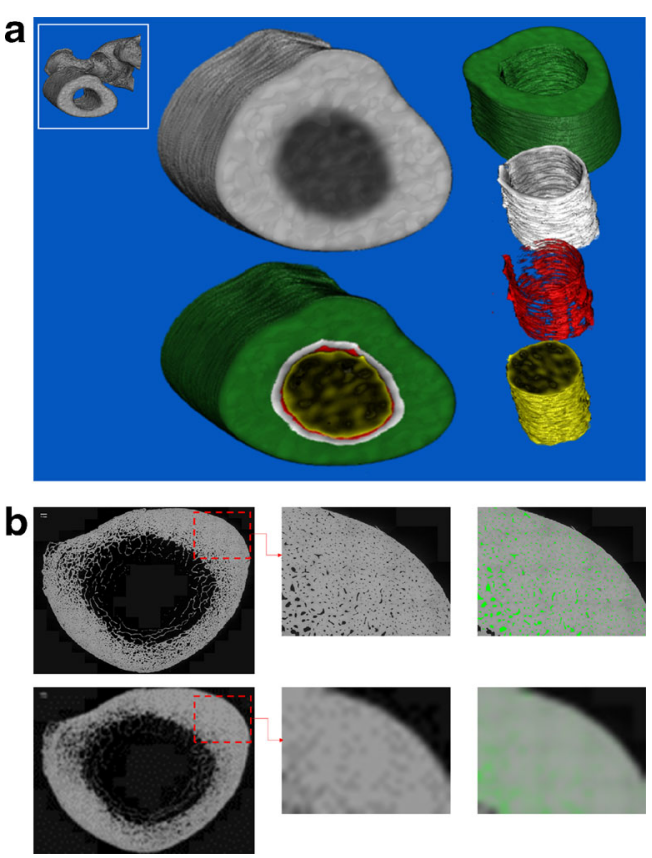

Fig. 1 a Segmented computed tomography image obtained at the femoral subtrochanter using non-threshold-based image analysis, showing the total cortical area (the area used for the cortical porosity measurements); consisting of the three cortical compartments: compact appearing cortex (green), the outer (white), and inner (red) transitional zones, and trabecular bone area (yellow). b Top left panel shows entire cross section in a 63-year-old woman (subtrochanteric region) acquired using scanning electron microscopy. Bottom Left panel shows the same cross section downsize to simulate a resolution of 740 microns. Top middle panel is magnified regions of interest (ROI) at 12.5 micron resolution pores are clearly visible. Bottom middle panel shows the same ROI but downsized to simulate a resolution of 740 microns. Pores are no longer visible, at least not as empty pixels. Right panels pores which are clearly visible have been color-coded green (top panel). Following the downsizing of the image to simulate a resolution of 740 microns (bottom panel), the presence of porosity in the image signaled by the green color is clearly visible although it does not appear as empty pixels

(Fig. 1a, b)). The porosity quantified by this algorithm is the proportion of emptiness within each voxel, or the fraction of the bone volume occupied by void (porosity). The size and number of pores were not determined by using this software. However, it is common practice to be able to accurately quantify structures or objects invisible to the naked eye. To measure porosity at the subvoxel level, below visibility, two referent attenuation values are required $\mathrm{P}$ : the background (muscle, water etc.) and B: the fully mineralized bone matrix (1200 $\left.\mathrm{mg} \mathrm{HA} / \mathrm{cm}^{3}\right)$. The proportion of the voxel volume occupied by mineralized bone matrix volume is its level of fullness (LOF). As previously reported, LOF of each voxel is estimated as $(\mathrm{LOF}) \%=\left(\mathrm{A}_{\mathrm{i}}-\mathrm{P}\right) /(\mathrm{B}-\mathrm{P})$, where $\mathrm{A}_{\mathrm{i}}$ is the attenuation of voxel $i$ [25]. From LOF, the void volume of each voxel or level of emptiness (porosity) $=100-$ LOF (\%) (Fig. 2). Accuracy of porosity measurements using $\mathrm{CT}$, voxel size 740 microns, was validated ex vivo by testing the agreement with HR-pQCT measurements, voxel size 82 microns, of the 
Fig. 2 To measure porosity, two referent attenuation values are required $P$ : the background (muscle, water etc.) and B: the fully mineralized bone matrix (1200 mg HA/ $\mathrm{cm}^{3}$ ). The proportion of the voxel volume occupied by mineralized bone matrix is its level of fullness (LOF) and is estimated as (LOF) $\%=\left(A_{i}-P\right) /(B-P)$, where $A_{i}$ is the attenuation of voxel $i$. From LOF, the void volume of each voxel or level of emptiness (porosity) $=100-$ LOF $(\%)$

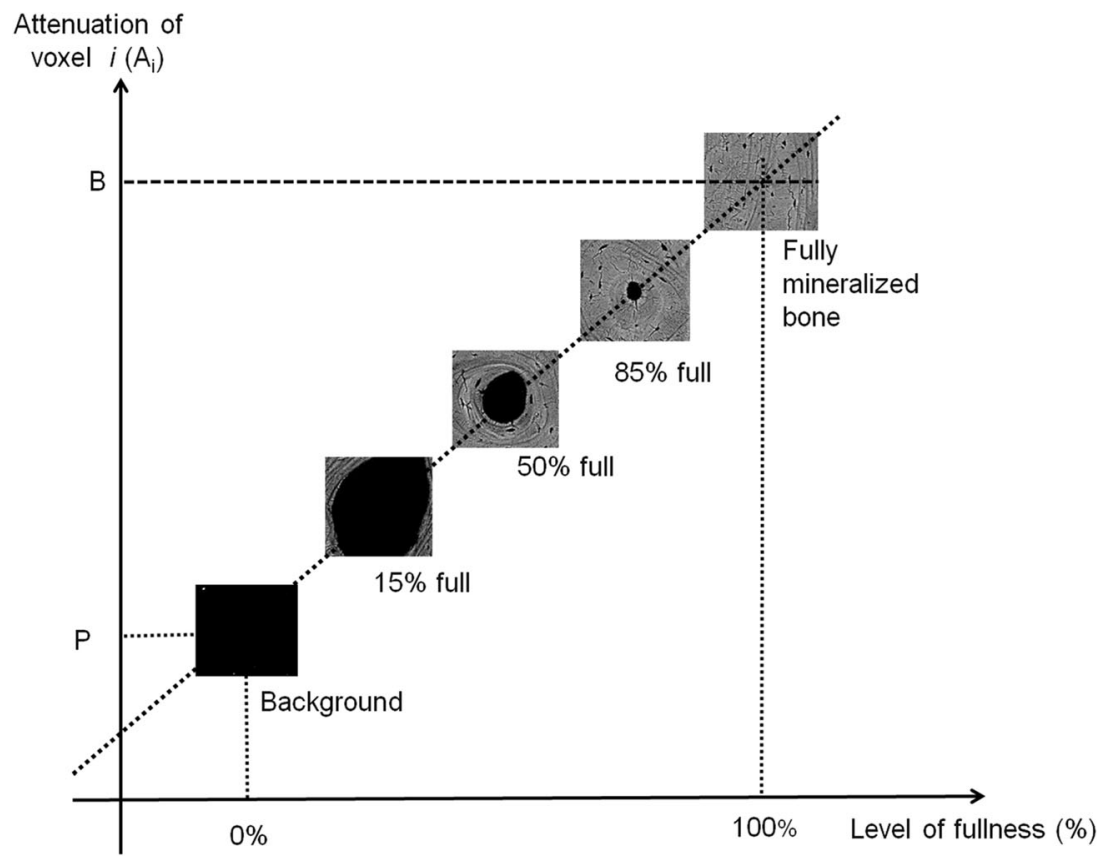

same ROI at the femoral subtrochanteric region in 5 cadaveric specimens. The correlation between porosity of the compact appearing cortex by these methods $\left(R^{2}\right)$ was 0.96 (Fig. 3a). The corresponding $R^{2}$ were $0.87,0.87$, and 0.94 for porosity of the outer and inner transitional zones, and the total cortex, respectively (Fig. 3b-d). As shown in the Bland-Altman plots (right panels), the error (difference between measurements by CT and HR-pQCT scanning) ranged from 0 to $10 \%$ depending a
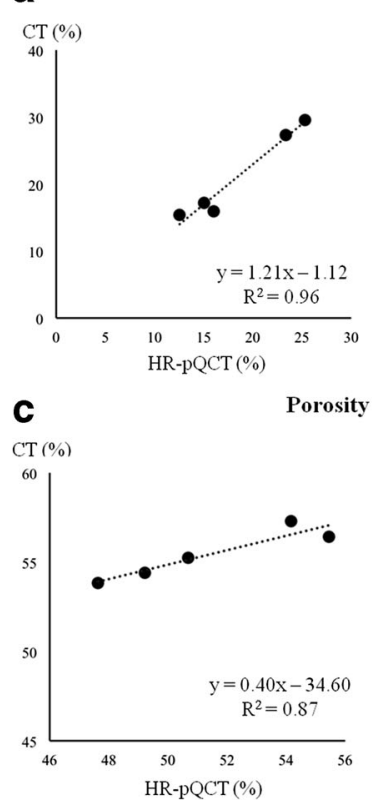

Porosity of compact-appearing cortex (\%)

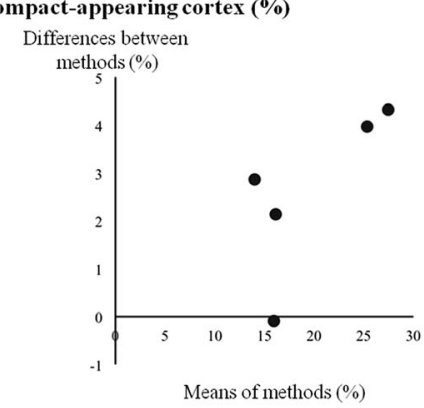

Porosity of inner transitional zone (\%)

$$
\text { Differences between }
$$

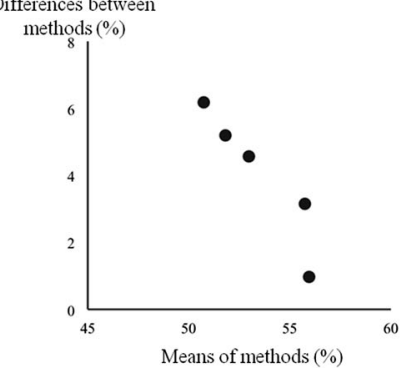

Fig. 3 Left panels the correlation between CT and gold standard (HR-pQCT) measurements of porosity of a the compact appearing cortex, $\mathbf{b}$ outer and $\mathbf{c}$ inner transitional zones, and $\mathbf{d}$ the total cortex at the femoral subtrochanter (all $p<0.05$ ). Right panel Bland-

on the compartment, and agreement between both measurements exceeded $90 \%$. Validation of the StrAx 1.0 software analyses of the femoral subtrochanter cortical porosity, as well as all standard CT parameters in this study, was performed by repositioning and rescanning a human hip phantom 10 times, and CVs were between 0.3 and $2.3 \%$. This human hip phantom was delivered with the CT scanner (Siemens Somatom Sensation 16, Erlangen, Germany).
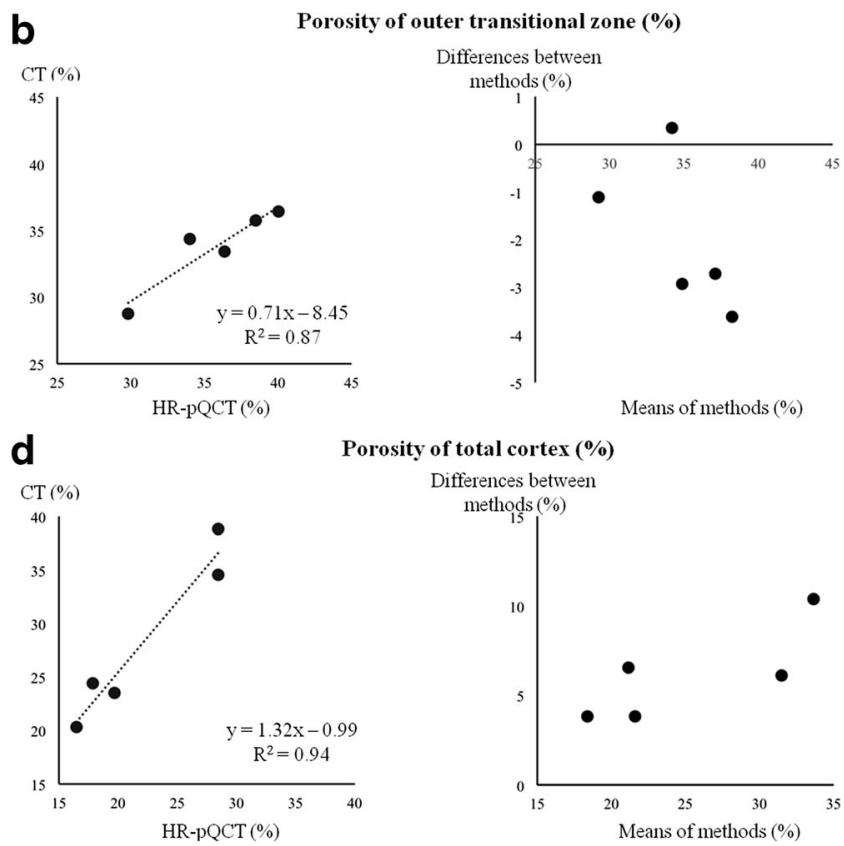

Altman plots of the error (difference between measurements by $\mathrm{CT}$ and HR-pQCT scanning) ranged from 0 to $10 \%$, and agreement between both measurements exceeded $90 \%$ 


\section{Statistical analyses}

All variables were normally distributed except the FRAX score. Log-transformation corrected the skewed distribution and did not change any results. Differences between cases and controls were assessed using analysis of variance and adjusted for age. Odds ratios (OR) with $95 \%$ confidence interval (CI) for fracture were calculated using logistic regression analyses, adjusted for age, height, weight, FN aBMD, and FRAX score and expressed per one SD difference and by threshold levels. To evaluate whether the association between cortical porosity and fracture was modified by FN aBMD or FRAX score, we included interaction terms. We explored sensitivity and specificity for fracture at selected thresholds for cortical porosity as the 75th, 80th, and 90th percentile. Using the thresholds for osteoporosis (FN aBMD T score $<-2.5$ ), FRAX score $>20 \%$, and cortical porosity $>80$ th percentile (based on all participants as reference), as potential criteria to consider intervention, we calculated the proportion of women in the fracture and control groups meeting these criteria, and the additional number of women identified when cortical porosity was included. These data are presented in Venn diagrams. Analyses were performed using SAS Software package, v9.3 (SAS Institute Inc., Cary, NC, USA) and STATA 12.0 (StataCorp, College Station, TX, USA) with $p<0.05$ considered significant.

\section{Results}

\section{FN aBMD, FRAX, cortical porosity, and fracture}

Fracture cases had lower total hip aBMD, lower FN aBMD, higher FRAX score, and higher femoral subtrochanteric cortical porosity than controls, all $p<0.001$ (Table 1). Each SD lower total hip aBMD (OR 2.21; 1.732.83), each SD lower FN aBMD (OR 2.11; 1.66-2.68), each SD higher FRAX-with-aBMD (OR 2.10; 1.64-2.70), each SD higher FRAX-without-aBMD (OR 1.62; 1.292.02 ), and each SD higher cortical porosity (OR 1.71; $1.38-2.11$ ) was associated with fracture (all $p<0.001$ ) (Fig. 4a).

\section{Cortical porosity is associated with fracture independent of FN aBMD and FRAX}

Each SD higher cortical porosity was associated with fracture independent of total hip aBMD (OR 1.30; $95 \%$ CI 1.02-1.64, $p=0.03$ ) in all women combined. Each SD higher cortical porosity was also associated with fracture independent of FN aBMD in all women combined (OR 1.39; 1.11-1.74, $p=0.005$, Fig. $4 \mathrm{a}$ ), in subgroups of women with normal FN aBMD (OR 1.53; 0.96-2.43), $p=0.07$, osteopenia (OR 1.32;
$1.00-1.75), p=0.055$, but not significantly in those with osteoporosis (OR 1.46; 0.57-3.71, $p=0.43$, Fig. 4b).

Similarly, cortical porosity was associated with fracture independent of FRAX-with-BMD in all women combined (OR 1.58; 1.27-1.97), $p<0.001$, in women with normal FN aBMD (OR 1.88; 1.21-2.94), $p=0.005$, osteopenia (OR 1.40; $1.06-1.85), p=0.02$, but not significantly in women with osteoporosis (OR 1.48; 0.68-3.23), $p=0.32$.

Cortical porosity was also associated with fracture independent of FRAX-without-BMD (OR 1.73; 1.40-2.15), $p<0.001$, Fig. $4 \mathrm{a}, \mathrm{b}$. There was no significant interaction between porosity, aBMD, FRAX-with-BMD, or FRAXwithout-BMD, all $p>0.20$. Further adjustment for oral corticosteroid use did not change the results.

\section{Fracture by porosity $>80$ th percentile, without or with osteoporosis, or FRAX $>20 \%$}

Of all women with fracture, $22 \%$ had normal FN aBMD, $69 \%$ osteopenia, and $9 \%$ osteoporosis (Table 1). Normal FN aBMD was protective (OR 0.29; 0.19-0.43, $p<0.001$ ), osteopenia was associated with fracture (OR 3.37; 2.16 $5.26, p<0.001)$, but osteoporosis was not significantly associated with fracture (OR 1.87; 0.86-4.07, $p=0.11$ ). In all women, independent associations with fracture were observed for porosity $>80$ th percentile (OR $2.70 ; 1.63-4.47$ ) and FRAXwith-BMD $>20 \%$ (OR 3.28; 1.77-6.08), both $p<0.001$.

In the subgroup of women with normal FN aBMD, high porosity ( $>80$ th percentile) was present in $21 \%$ of cases and $4 \%$ of controls and was associated with fracture (OR 6.96; $1.96-24.7, p=0.003)$. In the subgroup of women with osteopenia, high porosity was present in $30 \%$ of cases and $19 \%$ of controls and was associated with fracture, but did not achieve significance at $p<0.05$ level (OR 1.72; 0.94-3.13, $p=0.08$ ). In the subgroup of women with osteoporosis, high porosity was present in $39 \%$ of cases and $27 \%$ of controls but was not associated with fracture (OR 1.70; $0.33-8.67$, $p=0.53)$.

\section{Additional women with fracture identified by porosity than FN aBMD or FRAX}

Of all 211 fracture cases, only 18 women $(9 \%)$ were identified using the osteoporosis threshold, 45 women (21\%) using the FRAX-with-BMD threshold whereas high cortical porosity level identified 61 women (29\%), Fig. 5. Porosity identified $54(26 \%)$ women with fractures not identified by the osteoporosis threshold and $45(21 \%)$ women with fractures not identified by the FRAX-with-BMD threshold. Moreover, $115(55 \%)$ of fracture cases were not identified by any of these methods. In addition, FRAX-without-aBMD identified $56(27 \%)$ women with fractures. When porosity was measured, 41 (19 \%) additional women with fracture were 
Table 1 Characteristics in women with fractures and controls

\begin{tabular}{|c|c|c|c|}
\hline & Cases $(n=211)$ & Controls $(n=232)$ & $p$ value \\
\hline Age (years) & $68.4(7.7)$ & $68.3(6.7)$ & 0.94 \\
\hline Height $(\mathrm{cm})$ & $162.7(6.1)$ & $161.2(6.6)$ & 0.01 \\
\hline Weight $(\mathrm{kg})$ & $68.9(10.5)$ & $70.0(10.8)$ & 0.28 \\
\hline Total Hip aBMD $\left(\mathrm{mg} / \mathrm{cm}^{2}\right)$ & $854(113)$ & $930(115)$ & $<0.001$ \\
\hline Femoral neck (FN) aBMD $\left(\mathrm{mg} / \mathrm{cm}^{2}\right)$ & $794(100)$ & $860(112)$ & $<0.001$ \\
\hline Have normal FN aBMD, $n(\%)$ & $47(22.3)$ & $112(48.3)$ & $<0.001$ \\
\hline Have osteopenia, $n(\%)$ & $146(69.2)$ & $109(47.0)$ & $<0.001$ \\
\hline Have osteoporosis, $n(\%)$ & $18(8.5)$ & $11(4.7)$ & 0.11 \\
\hline FRAX score with FN aBMD (\%) & $15.1(8.4)$ & $10.8(4.9)$ & $<0.001$ \\
\hline FRAX score without FN aBMD (\%) & $16.3(10.2)$ & $12.7(6.2)$ & $<0.001$ \\
\hline \multicolumn{4}{|l|}{ Femoral subtrochanteric parameters } \\
\hline Total bone vBMD $\left(\mathrm{mg} \mathrm{HA} / \mathrm{cm}^{3}\right)$ & $684(113)$ & $750(90.0)$ & $<0.001$ \\
\hline Cortical vBMD $\left(\mathrm{mg} \mathrm{HA} / \mathrm{cm}^{3}\right)$ & $1025(72.6)$ & $1059(56.6)$ & $<0.001$ \\
\hline Cortical porosity $(\%)$ & $43.8(4.3)$ & $41.7(3.4)$ & $<0.001$ \\
\hline Trabecular bone volume/tissue volume (\%) & $0.27(0.02)$ & $0.27(0.02)$ & 0.81 \\
\hline Alcohol intake (drinks/week) & $2.7(2.5)$ & $2.8(2.3)$ & 0.80 \\
\hline Currently smoking, $n(\%)$ & $29(13.7)$ & $24(10.3)$ & 0.26 \\
\hline Physical activity (h/week) & $2.6(1.6)$ & $2.5(1.7)$ & 0.42 \\
\hline History of previous fracture, $n(\%)$ & $54(25.6)$ & 0 & \\
\hline Parental history of hip fracture, $n(\%)$ & $34(16.1)$ & $37(16.0)$ & 0.96 \\
\hline Self-reported excellent/good health status, $n(\%)$ & $147(70.3)$ & $165(71.1)$ & 0.86 \\
\hline Rheumatoid arthritis, $n(\%)$ & $11(5.2)$ & $8(3.5)$ & 0.36 \\
\hline Oral corticosteroid use, $n(\%)$ & $8(3.8)$ & $2(0.9)$ & 0.04 \\
\hline Take calcium supplements, $n(\%)$ & $44(20.9)$ & $28(12.1)$ & 0.01 \\
\hline Take supplements of vitamin $\mathrm{D}, n(\%)$ & $163(77.3)$ & $166(71.6)$ & 0.17 \\
\hline
\end{tabular}

Data are mean (SD) or number (\%)

$p$ values are adjusted for age using analysis of variance

FRAX Fracture Risk Assessment Tool for calculation of the 10-year probability of a major osteoporotic fracture, $a B M D$ areal bone mineral density, $\nu B M D$ volumetric bone mineral density identified over that identified by FRAX-without-aBMD alone. Of 232 controls, osteoporosis was present in $5 \%$, high FRAXwith-BMD in $7 \%$, and high cortical porosity in $12 \%$ (Fig. 5). The sensitivity at these thresholds for osteoporosis, FRAXwith-BMD, and cortical porosity was 9, 21, and $29 \%$, and specificity was 95,93 , and $88 \%$ (Table 2 ). When porosity $>80$ th percentile was combined with FRAX-with-BMD $>20 \%$, the sensitivity for fracture was $43 \%$ and specificity was $83 \%$.

\section{Discussion}

We report that cortical porosity of the proximal femur was associated with nonvertebral fractures in postmenopausal women independent of aBMD and FRAX score. This was observed in women with normal aBMD and osteopenia, persons commonly regarded as being at low fracture risk. Porosity was not significantly associated with fracture in women with osteoporosis. Measurement of porosity identified 26 and $21 \%$ additional women with fracture than identified by the osteoporosis or FRAX thresholds, respectively.

Women concerned about bone fragility but found to have aBMD in the normal and osteopenic range would often be reassured and not offered therapy even though most fractures arise from this large segment of the population [4]. The observations reported here address this unmet need. Measurement of porosity is likely to improve identification of women with normal BMD or osteopenia at risk for fracture as porosity is likely to capture fracture risk not captured by the aBMD measurement.

This report suggests that proximal femoral cortical porosity measured from clinical CT images in vivo identifies $26 \%$ additional fracture cases over that identified by FN aBMD T-score $<-2.5$. Several studies demonstrate that measurement of porosity is associated with fractures and that women with osteopenia with fractures have higher cortical porosity. Most of these studies have used HR-pQCT, a method available in 
Fig. 4 a Odds ratio (OR) and $95 \%$ confidence interval (CI) for nonvertebral fracture per each standard deviation (SD) lower in femoral neck (FN) areal bone mineral density (aBMD), each SD higher in fracture risk assessment tool (FRAX) score with BMD, each SD higher in FRAX score without BMD, each SD higher in cortical porosity, in all women. b OR $(95 \% \mathrm{CI})$ for nonvertebral fracture per each SD higher cortical porosity in women with normal femoral neck areal bone mineral density (FN aBMD, $n=159,47$ with fracture), osteopenia $(n=255,146$ with fracture) and osteoporosis $(n=29,18$ with fracture $)$
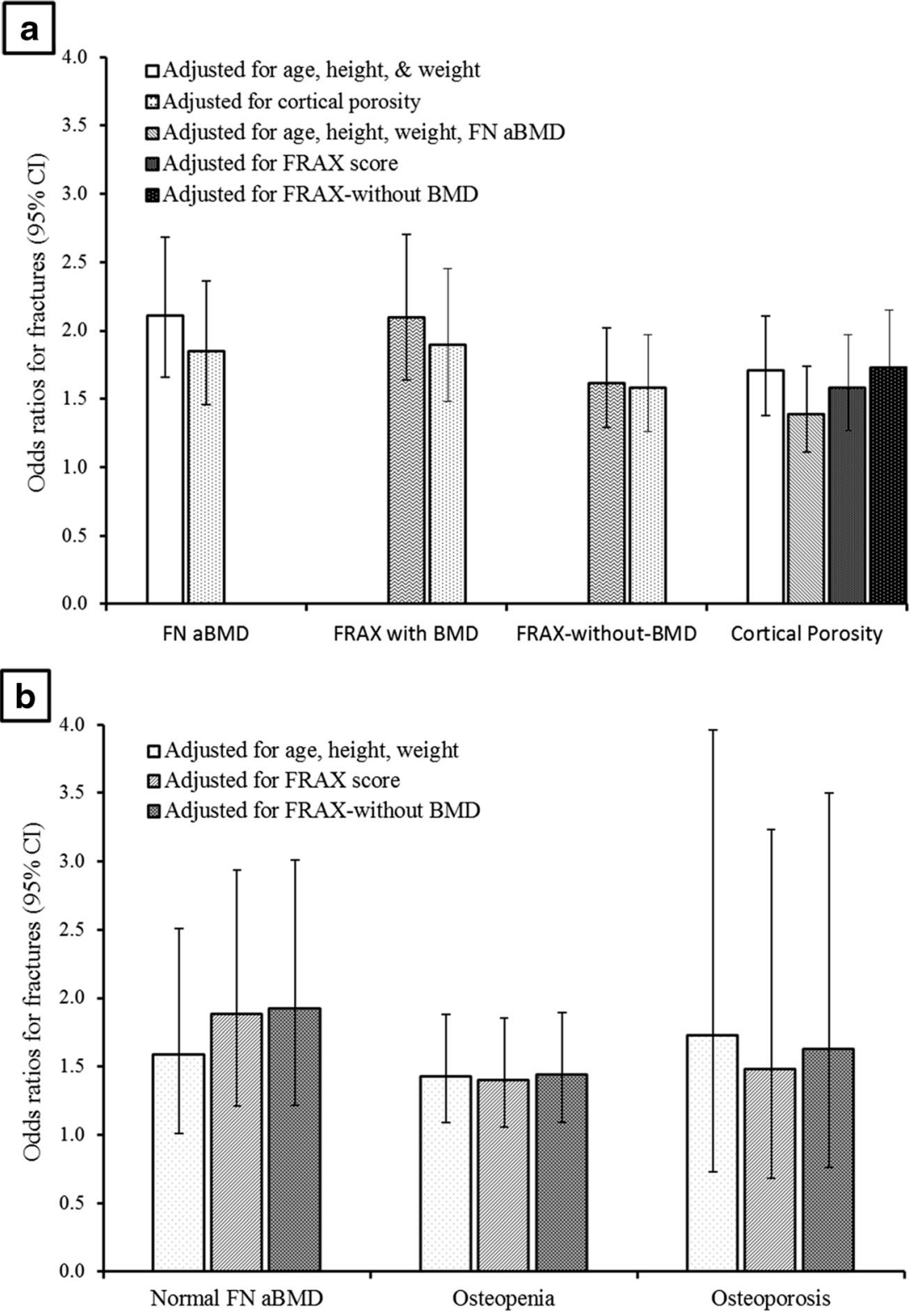

only a few centers around the world [18, 20, 26]. We were unable to show an association between cortical porosity and fracture in the small group of women with osteoporosis, due to lack of statistical power. However, most of the women with osteoporosis at the femoral neck did not have high porosity. Further studies are needed to examine this question.

FRAX score was associated with fracture as in other cohorts [6-8]. However, the measurement of porosity was associated with fracture independent of FRAX, and identified $21 \%$ additional women with fracture over that identified by FRAX-with-BMD threshold $>20 \%$. In settings where bone densitometry is not available, CT is usually available, and rather than using FRAX-without-BMD alone, combining this with porosity assessment appears to identify $19 \%$ more fracture cases than identified by FRAX-without-BMD threshold $>20 \%$.

Although measurement of porosity improved sensitivity for fracture by identifying additional women with fracture than identified using aBMD threshold for osteoporosis or the FRAX threshold, $55 \%$ of the fracture cases were not identified by any of these methods. Thus, improving sensitivity remains a challenge that may be met by measurement of other material and structural propertied to build an architectural risk score and by using fall characteristics. The present results do not negate the role of trabecular bone in bone fragility. Studies using HR-pQCT of the distal radius and tibia have reported trabecular vBMD associated with fracture independent of aBMD, while one study using CT of the proximal femur reported that only cortical, not trabecular, vBMD remained 

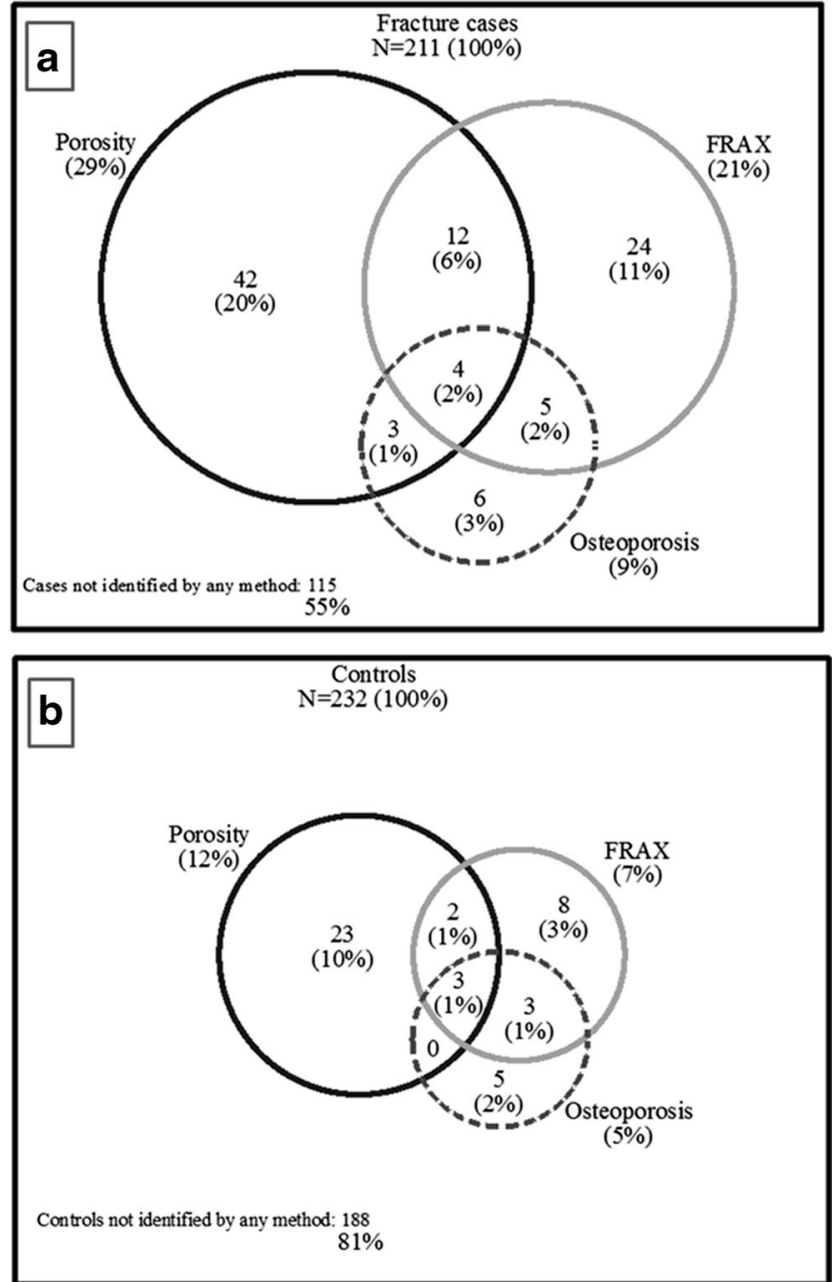

Fig. 5 Venn diagrams illustrate the number and proportion of women identified using threshold for osteoporosis (femoral neck areal bone mineral density (BMD) T-score $<-2.5$ ), fracture risk assessment tool (FRAX) score with-BMD $>20 \%$, and cortical porosity $>80$ th percentile, $\mathbf{a}$ in 211 women with fracture and $\mathbf{b}$ in 232 controls

associated with hip fracture after accounting for aBMD [27-29]. Another study using CT of the proximal femur reported both cortical and trabecular vBMD associated with hip fractures [30].
In this study, the aBMD using DXA was measured at the femoral neck, while cortical porosity was measured at the subtrochanteric region. These two sites are located near each other, but there are huge differences in site-specific biology as one site is rich in trabecular bone with a variable thickness of cortex around its perimeter, the other site consist of a thick cortex and little trabecular bone. Better understanding of this site-specific biology may improve the use of cortical porosity as an indicator of fracture risk for certain individuals.

A strength of this nested case-control study is that it is based on a general population and a validated fracture registry [31]. The newly developed StrAx software for quantification of bone architecture is validated by confirming strong correlation between measurements using CT scans and HR-pQCT, the method which has been used for previous measurements of bone architecture, and by rescanning a human hip phantom using the same standard CT machines as used for the participants of the study with good reproducibility. The measurements were obtained from the proximal femur, a site of the most serious and common fragility fracture.

The study has several limitations. The retrospective casecontrol design may have introduced selection bias and the index fractures occurred at a median of 6.6 years before the women had their CT scans for porosity assessment. As most of the cases had wrist fractures, we showed that cortical porosity of the proximal femur is associated with wrist fracture but it remains to study how cortical porosity is associated hip fracture.

In conclusion, cortical porosity at the proximal femur is a quantifiable risk factor for fracture that captures additional elements of bone strength not captured by aBMD or FRAX. A measurement of porosity improves identification of women with nonvertebral fracture when combined with aBMD or FRAX thresholds. Measurement of porosity is likely to better identify women at increased risk for fractures and to be a clinically useful tool that helps target treatment to those in need and avoid unnecessary treatment of those who do not need it. Further research will be needed to determine the association of subtrochanteric cortical porosity with fracture risk

Table 2 Sensitivity and specificity for fractures at selected thresholds

\begin{tabular}{|c|c|c|c|c|}
\hline & Sensitivity $\%$ & $95 \% \mathrm{CI}$ & Specificity \% & $95 \% \mathrm{CI}$ \\
\hline Femoral neck aBMD T-score below $-2.5 \mathrm{SD}$ & 8.5 & $5.1-13.1$ & 95.3 & $91.7-97.6$ \\
\hline FRAX-with-aBMD $>20 \%$ & 21.3 & $16.0-27.5$ & 93.1 & $89.0-96.0$ \\
\hline FRAX-without-aBMD > $20 \%$ & 25.1 & $19.4-31.5$ & 91.8 & $87.5-95.0$ \\
\hline Cortical porosity $>75$ th percentile & 33.6 & $27.3-40.5$ & 83.2 & $77.7-87.8$ \\
\hline Cortical porosity $>80$ th percentile & 28.9 & $22.9-35.5$ & 87.9 & $83.0-91.8$ \\
\hline Cortical porosity $>90$ th percentile & 16.1 & $11.4-21.8$ & 95.3 & $92.2-97.9$ \\
\hline FRAX-with-aBMD or porosity $>80$ th percentile & 42.7 & $35.9-49.6$ & 83.2 & $77.7-87.8$ \\
\hline FRAX-without-aBMD or porosity $>80$ th percentile & 44.5 & $37.7-51.5$ & 80.6 & $74.9-85.5$ \\
\hline
\end{tabular}

FRAX Fracture Risk Assessment Tool for calculation of the 10-year probability of a major osteoporotic fracture, $a B M D$ areal bone mineral density 
in a prospective population study, to improve the sensitivity using other material and structural abnormalities that contribute to fracture risk, to determine treatment threshold for cortical porosity and how to best treat those with higher porosity, as they may need to be treated differently than those individuals with lower aBMD or higher FRAX score.

\begin{abstract}
Acknowledgments The North Norwegian Health Authorities funded the study (ID 5645 1002-11, ID 9167/SFP1090-13, ID 9168/SFP1135-13) but had no role in design and conduct of the study; in the collection, analyses, and interpretation of the data; or in the preparation, review, or approval of the manuscript. The Tromsø Study provided access to data, staff at the Department of Research at the University Hospital of North Norway (UNN) recruited women, staff at the Department of Radiology and Department of Radiation, UNN scanned the patients, organized the radiation procedures and the CT images, and StraxCorp, Melbourne analyzed the CT images.
\end{abstract}

Conflicts of interest EFE reported that his research has been supported by Amgen, Novartis, Eli Lilly, and IDS, RZ has received consulting fees/ lecture fees/grant support from Amgen, MSD, Servier. ES have received consulting fees/lecture fees/grant support from Amgen, MSD, Novartis, Sanofi Aventis, Servier. ÅB has received lecture fees from Eli Lilly. RZ and ES are inventors of the StrAx method and directors of StraxCorp. AGZ is one of the inventors of StrAx 1.0. LAA, RS, RMJ, OPE, and YB declare that they have no conflict of interest.

Open Access This article is distributed under the terms of the Creative Commons Attribution-NonCommercial 4.0 International License (http:// creativecommons.org/licenses/by-nc/4.0/), which permits any noncommercial use, distribution, and reproduction in any medium, provided you give appropriate credit to the original author(s) and the source, provide a link to the Creative Commons license, and indicate if changes were made.

\section{References}

1. Cummings SR, Melton LJ (2002) Epidemiology and outcomes of osteoporotic fractures. Lancet 359:1761-1767

2. Kanis JA, Oden A, Johnell O, De Laet C, Jonsson B, Oglesby AK (2003) The components of excess mortality after hip fracture. Bone $32: 468-473$

3. Kanis JA (1994) Assessment of fracture risk and its application to screening for postmenopausal osteoporosis: synopsis of a WHO report. Osteoporos Int 4:368-381

4. Siris ES, Chen YT, Abbott TA, Barrett-Connor E, Miller PD, Wehren LE, Berger ML (2004) Bone mineral density thresholds for pharmacological intervention to prevent fractures. Arch Intern Med 164:1108-1112

5. Schuit SC, van der Klift M, Weel AE, de Laet CE, Burger H, Seeman E, Hofman A, Uitterlinden AG, van Leeuwen JP, Pols HA (2004) Fracture incidence and association with bone mineral density in elderly men and women: the Rotterdam Study. Bone 34: 195-202

6. Kanis J, Johnell O, Oden A, Johansson H, McCloskey E (2008) FRAX $^{\mathrm{TM}}$ and the assessment of fracture probability in men and women from the UK. Osteoporos Int 19:385-397

7. Leslie WD, Brennan SL, Lix LM, Johansson H, Oden A, McCloskey E, Kanis JA (2013) Direct comparison of eight national FRAX(R) tools for fracture prediction and treatment qualification in Canadian women. Arch Osteoporos 8:145
8. Johansson H, Kanis JA, Oden A, Johnell O, McCloskey E (2009) $\mathrm{BMD}$, clinical risk factors and their combination for hip fracture prevention. Osteoporos Int 20:1675-1682

9. Schaffler MB, Burr DB (1988) Stiffness of compact bone: effects of porosity and density. J Biomech 21:13-16

10. van der Linden JC, Weinans H (2007) Effects of microarchitecture on bone strength. Curr Osteoporos Rep 5:56-61

11. Zebaze RM, Ghasem-Zadeh A, Bohte A, Iuliano-Burns S, Mirams M, Price RI, Mackie EJ, Seeman E (2010) Intracortical remodelling and porosity in the distal radius and post-mortem femurs of women: a cross-sectional study. Lancet 375:1729-1736

12. Hernlund E, Svedbom A, Ivergard M, Compston J, Cooper C, Stenmark J, McCloskey EV, Jonsson B, Kanis JA (2013) Osteoporosis in the European Union: medical management, epidemiology and economic burden. A report prepared in collaboration with the International Osteoporosis Foundation (IOF) and the European Federation of Pharmaceutical Industry Associations (EFPIA). Arch Osteoporos 8:136

13. Bell KL, Loveridge N, Power J, Garrahan N, Stanton M, Lunt M, Meggitt BF, Reeve J (1999) Structure of the femoral neck in hip fracture: cortical bone loss in the inferoanterior to superoposterior axis. J Bone Miner Res 14:111-119

14. Bell KL, Loveridge N, Power J, Garrahan N, Meggitt BF, Reeve J (1999) Regional differences in cortical porosity in the fractured femoral neck. Bone 24:57-64

15. Bell KL, Loveridge N, Jordan GR, Power J, Constant CR, Reeve J (2000) A novel mechanism for induction of increased cortical porosity in cases of intracapsular hip fracture. Bone 27:297-304

16. Zebaze RM, Libanati C, Austin M et al (2014) Differing effects of denosumab and alendronate on cortical and trabecular bone. Bone 59:173-179

17. Bala Y, Chapurlat R, Cheung AM et al (2014) Risedronate slows or partly reverses cortical and trabecular microarchitectural deterioration in postmenopausal women. J Bone Miner Res 29:380-388

18. Bjørnerem Å, Bui QM, Ghasem-Zadeh A, Hopper JL, Zebaze R, Seeman E (2013) Fracture risk and height: an association partly accounted for by cortical porosity of relatively thinner cortices. J Bone Miner Res 28:2017-2026

19. Dhainaut A, Hoff M, Syversen U, Haugeberg G (2013) Cortical hand bone porosity and its association with distal radius fracture in middle aged and elderly women. PLoS One 8:e68405

20. Bala Y, Zebaze R, Ghasem-Zadeh A et al (2014) Cortical porosity identifies women with osteopenia at increased risk for forearm fractures. J Bone Miner Res 29:1356-1362

21. Jacobsen BK, Eggen AE, Mathiesen EB, Wilsgaard T, Njolstad I (2012) Cohort profile: the Tromsø Study. Int J Epidemiol 41:961-967

22. Ahmed LA, Center JR, Bjørnerem $\AA$ et al (2013) Progressively increasing fracture risk with advancing age after initial incident fragility fracture: the Tromsø study. J Bone Miner Res 28:2214-2221

23. Bjørnerem Å, Ahmed LA, Jørgensen L, Størmer J, Joakimsen RM (2011) Breastfeeding protects against hip fracture in postmenopausal women: the Tromsø study. J Bone Miner Res 26:2843-2850

24. Emaus N, Omsland TK, Ahmed LA, Grimnes G, Sneve M, Berntsen GK (2009) Bone mineral density at the hip in Norwegian women and men-prevalence of osteoporosis depends on chosen references: the Tromsø Study. Eur J Epidemiol 24: 321-328

25. Zebaze R, Ghasem-Zadeh A, Mbala A, Seeman E (2013) A new method of segmentation of compact-appearing, transitional and trabecular compartments and quantification of cortical porosity from high resolution peripheral quantitative computed tomographic images. Bone 54:8-20

26. Nishiyama KK, Macdonald HM, Buie HR, Hanley DA, Boyd SK (2010) Postmenopausal women with osteopenia have higher cortical porosity and thinner cortices at the distal radius and tibia than 
women with normal aBMD: an in vivo HR-pQCT study. J Bone Miner Res 25:882-890

27. Melton LJ 3rd, Riggs BL, van Lenthe GH, Achenbach SJ, Muller R, Bouxsein ML, Amin S, Atkinson EJ, Khosla S (2007) Contribution of in vivo structural measurements and load/strength ratios to the determination of forearm fracture risk in postmenopausal women. J Bone Miner Res 22:1442-1448

28. Sornay-Rendu E, Boutroy S, Munoz F, Delmas PD (2007) Alterations of cortical and trabecular architecture are associated with fractures in postmenopausal women, partially independent of decreased BMD measured by DXA: the OFELY study. J Bone Miner Res 22:425-433
29. Yang L, Udall WJ, McCloskey EV, Eastell R (2014) Distribution of bone density and cortical thickness in the proximal femur and their association with hip fracture in postmenopausal women: a quantitative computed tomography study. Osteoporos Int 25:251263

30. Cheng X, Li J, Lu Y, Keyak J, Lang T (2007) Proximal femoral density and geometry measurements by quantitative computed tomography: association with hip fracture. Bone 40:169-174

31. Joakimsen RM, Fønnebø V, Søgaard AJ, Tollan A, Størmer J, Magnus JH (2001) The Tromsø Study: registration of fractures, how good are self-reports, a computerized radiographic register and a discharge register? Osteoporos Int 12:1001-1005 